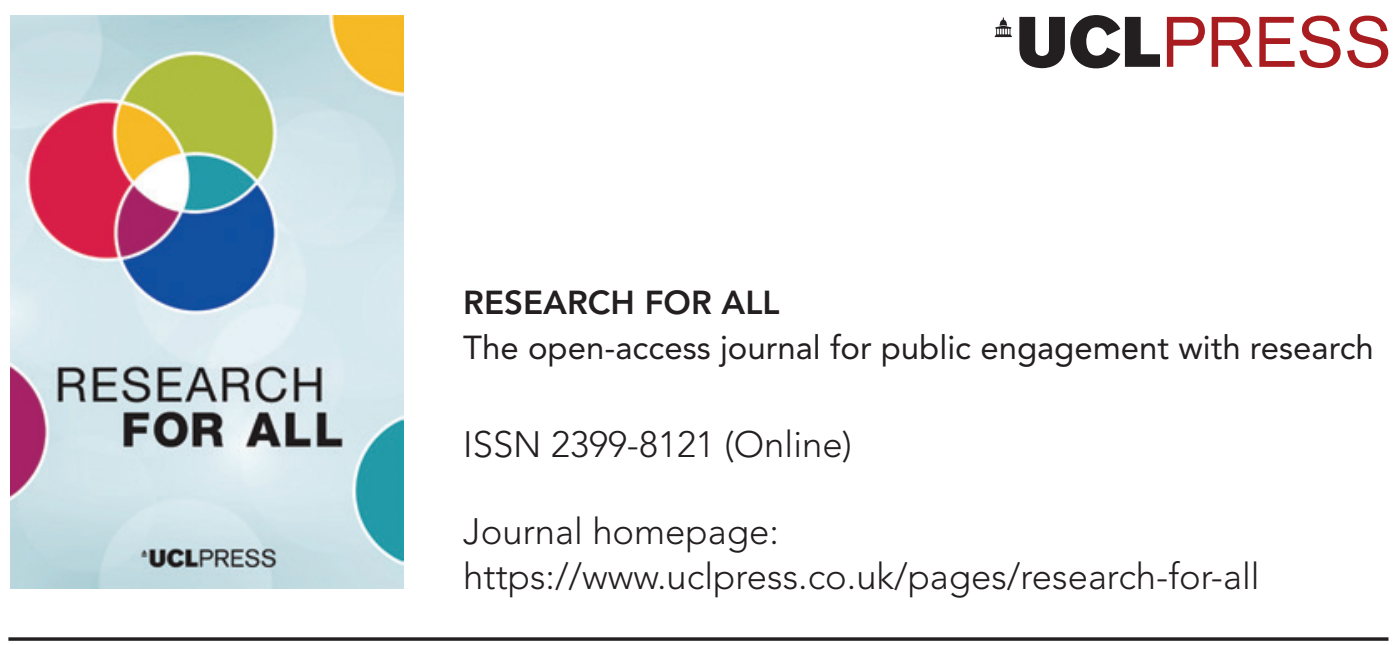

\title{
Reflections from the field: Researchers' experiences of co-production
}

Jenny Barke D, Helen Thomas-Hughes (D) and Marilyn Howard (D)

\section{How to cite this article}

Barke, J., Thomas-Hughes, H. and Howard, M. (2020) 'Reflections from the field: Researchers' experiences of co-production'. Research for All, 4 (2), 169-79. Online. https://doi.org/10.14324/RFA.04.2.03

Submission date: 14 May 2019

Acceptance date: 20 April 2020

Publication date: 22 September 2020

\section{Peer review}

This article has been peer-reviewed through the journal's standard double-blind peer review, where both the reviewers and authors are anonymized during review.

\section{Copyright}

(C) 2020 Barke, Thomas-Hughes and Howard. This is an open-access article distributed under the terms of the Creative Commons Attribution Licence (CC BY) 4.0 https://creativecommons.org/ licenses/by/4.0/, which permits unrestricted use, distribution and reproduction in any medium, provided the original author and source are credited.

\section{Open access}

Research for All is a peer-reviewed open-access journal. 


\title{
Reflections from the field: Researchers' experiences of co-production
}

\author{
Jenny Barke*, Helen Thomas-Hughes and Marilyn Howard - University of \\ Bristol, UK
}

\begin{abstract}
This paper draws on conversations between a group of research associates who worked on a large-scale co-produced research project, Productive Margins: Regulating for Engagement. Through our conversations and subsequent analysis, three themes emerged regarding our experiences working on the project: (1) Working across difference; (2) Engaging with arts practice; and (3) Creating the conditions for co-production. This paper reflects on these themes, and we suggest that there is a need to focus on and explore the processes of co-producing research in terms of pragmatics and epistemology.
\end{abstract}

Keywords: co-production, participatory research, community engagement, community researchers, arts-based research

\section{Key messages}

- Ensure that everyone involved in a collaborative project has a shared understanding of its aims and workings.

- Be clear about how and why arts-based methods are employed in a project.

- Take time to develop relationships and understand why people are involved in a project and what outcomes are important to them.

\section{Introduction}

In the UK, 'co-production' refers to collaborative and participatory research approaches that seek to challenge knowledge hierarchies and redistribute social power within the research process (Facer and Enright, 2016). Co-production aims to challenge powerful majority perspectives (Beebeejaun et al., 2015), and to stimulate transformational outcomes (Heron and Reason, 2008) motivating progressive social change in communities (Banks et al., 2014). The idea of co-producing research draws on highly diverse traditions of participatory, action, collaborative and communityengaged research (Facer and Enright, 2016). As such, co-production can be a difficult methodology to define (Thomas-Hughes, 2018).

Productive Margins: Regulating for Engagement was a collaboration between the University of Bristol (in South West England), Cardiff University (in South Wales) and seven community organizations in these two geographic areas. The aim of the programme was for community organizations and academics to co-produce research projects with community members, exploring how communities at the margins might reimagine and engage in processes of regulation that impact on everyday lives 
(McDermont et al., 2020). Howard et al. (2020: 1) explain that regulation 'permeates everyday life and is yet almost invisible'. The programme defined regulation as oriented towards process (rather than outcomes); seeking behaviour change; where there are rules of the game; where both regulator and those who are regulated have agency in the process; and enabling innovation. Regulation means different things to different people, and working groups within Productive Margins defined regulation depending on the topic of their project, and the academics and community partners involved. For instance, the project about loneliness attended to alternative regulatory systems for knowledge production, while the project exploring food defined regulation as the invisible rules that influence food production and consumption (see McDermont et al., 2020).

A central feature of the programme was the Productive Margins Research Forum, which was not a conventional advisory board, but rather a site of experimentation, acting as a dynamic location for co-producing knowledges, enhancing exchange and dissemination, and developing innovative methods for the social sciences, arts and humanities (Cohen et al., 2020). Within this forum, individual projects were developed that explored a range of topics, including: food habits, Muslim community engagement, the loneliness of older people and life on a low income. Projects came online at different times over the lifespan of Productive Margins. Each project was managed by a working group that was comprised of academics and representatives from community organizations. As projects emerged, research associates were employed and reported to the working groups. Research associates came from academic and community development backgrounds and had varying degrees of experience working in research. In three of the projects, research associates were employed directly by community organizations; this was a conscious attempt to redistribute some of the programme resources away from the universities. In three other projects, the research associates were employed by the university.

Community co-researchers were involved in individual projects in quite different ways. Some projects recruited community members to work specifically on the project, provided training and worked with community co-researchers to develop research questions, carry out research activities, and analyse and disseminate findings. Other projects worked with existing community groups and took an action research approach, working with community co-researchers to explore an aspect of their experience. In another example, initial research was carried out by an academic researcher and artists, and findings were presented to the local community, leading to several groups becoming involved in exploring findings and thinking about 'solutions'. (See McDermont et al., 2020 for accounts of individual projects.)

As the individual projects completed, a group of research associates expressed an interest in meeting to discuss their experiences across the programme. To support this, Productive Margins organized two writing retreats during which we took part in group discussions, one-to-one interviews and co-writing activity. This paper is based on conversations between six researchers, all of whom are white, speak English as a first language and are female. Our ages ranged from 20s to 40s. Interviews and group discussions were analysed explicitly to explore issues around quality in co-production (Howard and Thomas-Hughes, 2020) and community researcher training (ThomasHughes and Barke, 2018).

As a group, we identified several cross-cutting issues that we felt warranted further reflection. In this paper, we aim to open up a reflexive space in which some of the ideological and practical challenges of undertaking co-produced research can be elucidated. To do this, we have defined three central themes from our discussions to 
reflect on our own co-produced research and the challenges that we have encountered within our day-to-day practice.

\section{Recurring themes}

The first theme, 'Working across difference', relates to recognizing, valuing and understanding the different types of expertise and knowledges involved in coproduction. The second theme explores another area we found ourselves coming back to across our conversations: the role and practice of arts-based methods in coproduced research. As a group, we had very different experiences of the processes and outcomes of employing these methods and working with artists. The third theme running across our discussions relates to the idea of 'serendipity', which can be defined as the occurrence and development of events by chance. We agreed that in our experience there was little serendipity (or chance) in the process of co-producing research, but rather a lot of hard work in fostering relationships and supporting group cohesion to create the conditions for co-production.

\section{Working across difference}

Co-producing research comes from a desire to include a range of diverse voices with different types of expertise within the research process. This necessitates developing practices that support and promote participation by all parties (Beebeejaun et al., 2014). It is critical that within this, no one type of expertise should be privileged over another (Pohl et al., 2010). It is also imperative that the integrity or rigour of the research process is maintained to produce good-quality and impactful co-produced research (Armstrong and Alsop, 2010).

We all felt that the delicate balancing act of engaging with lived experience, academic and professional knowledge and expertise, while mainlining the rigour of the research process was largely held by us as research associates. We were often the only team member who engaged with all collaborators and had the most time allocated to the project, so this was often a pragmatic issue. At times we all struggled with how to work across this difference, and several research associates described uncertainty regarding how to manage the conflict to which this difference could lead. In one project, this conflict related to community members, partners and academics holding quite different ideas of what the project's aim, and remit, was. In another project, academics and community members and partners struggled to agree on what constituted data and how they would be managed. In both examples, all collaborators were employing their expertise and working within their institutional systems. Negotiating a compromise involved creative solutions, conversations outside collective spaces and a great deal of trust in one another. We reflected that this relied on us developing strong relationships with all groups, so that we could listen, understand and attend carefully to different partners and develop compromises, solutions and plans that all could recognize and work with.

We all felt that it was important to acknowledge that working with different groups and organizations involves working across systems that may be significantly different, operating to different timescales that may be challenging to align. While there can be negotiations regarding many aspects of collaborative research projects, there are also certain structures, such as university ethics or community organization data management plans and volunteer policies, which must be adhered to. It is essential to have conversations early on regarding project management. If systems are not discussed and time frames made clear, misunderstandings occur. For example, the 
process of bidding for, gaining funding and setting up projects is often much longer within university frameworks than in community organizations. On one project, as soon as the research was defined, the community partner set up a group ready to engage with the project; when it then took almost a year to have all the funding and personnel in place, the community partner felt frustrated, and the group that had been formed had moved in a different direction to the research. At other points, we faced challenges regarding community groups being ready to go with research activity but us needing to hold them back while we negotiated ethics procedures and university processes. On one project, there was a significant difference between the sorts of activities that different community organizations considered to be research, with concerns from one community organization that research project funds were being used to supplement the existing community development activities of another community organization under the guise of 'research'. This caused tension between community partners. There were also occasions when community organizations needed proposals or written material to be reviewed by particular people, or needed branding added. On several occasions, this delayed sending information out and deadlines were missed. The impact of these issues could have been mitigated had there been a clear conversation upfront about how systems worked, what timelines might look like, and what research activities might be. We felt that greater clarity would have allowed us to develop project-level systems and plans that supported different institutional systems.

In discussing the importance of including different types of expertise within coproduced research, we wrestled with questions about the way in which universities hold power, and how they distribute or withhold it. We agree with Porter (2010) that co-produced research should challenge the embedded knowledge hierarchies of the expert versus the layperson. Universities are in a position of power in co-produced research that needs to be acknowledged: they are positioned as the experts in research, they have often secured and hold the funding for any project, and it is likely that an academic will be the project lead. Co-producing research involves not privileging academic knowledge and systems, while often working within academic frameworks and funding regimes. We reflected that as research associates in the early career stage this can be challenging to navigate (Enright and Facer, 2017) - there is an explicit need for us to 'fit in to' academic structures. Negotiating academic systems and developing innovative practice to challenge embedded knowledge hierarchies can be precarious for early career researchers on short-term contracts. We suggest that early career researchers on collaborative co-produced projects would benefit from practical institutional support and training, as well as mentoring.

Across the Productive Margins programme, there was an explicit commitment to acknowledge and promote different kinds of knowledge to address power imbalances. Productive Margins aimed to distribute power by developing projects collaboratively, through forums and working groups that were comprised of community professionals and academics. This provided an exciting space in which we were able to explore diverse methods and knowledges. However, we wondered if at times there was a reluctance from some academics to voice their opinions and knowledge. We wondered if a concern about being perceived as part of the 'powerful university' led to the withdrawal of useful and insightful academic knowledge and expertise. Our wonderings were compounded when, towards the end of the programme, some community partners mused as to why academics with particular knowledge specialisms had not been more forthcoming in the early stages of projects. It is certainly important that academics create space for other forms of expertise to be heard. However, it is also important that they contribute their knowledge appropriately. On a few of the 
projects, research associates felt that research had struggled to get off the ground in the initial stages as some academic leads did not want to appear overbearing. At times, we felt that projects needed additional steering and guidance. One community lead became quite frustrated with this, feeling that some academics were deliberately withholding their expertise, which they felt wasted valuable project time. Setting up a research project is something that benefits from academic support. If academics simply withdraw, not wanting to appear overbearing, then a void appears, and the project can lose an important aspect.

Those of us who worked with community co-researchers reflected that the challenges of involving community members in research should not be underestimated, and, as Burholt et al. (2010) suggest, there is a need for rigorous methods to ensure meaningful involvement. For community members without a research background, training can be an essential prerequisite to involvement in fieldwork; otherwise, people may put themselves or others in danger, or may cause distress (Boynton, 2002). Training can create an opportunity where those taking part in a research project can collaboratively develop an understanding of what is being asked of them, and build the skills to carry out research tasks within an agreed system and an ethical framework. Training is also a vehicle for defining a project and getting to know one another. We felt that it was during training that we really got to know and understand the knowledge and expertise of the community co-researchers. This was essential to developing research plans that were genuinely built on the knowledge and expertise that community co-researchers brought to the project (Barke, 2017a,b).

Reflecting on how we worked across difference and brought together different institutional systems, knowledge and expertise, we agreed that it was essential to take time to develop and maintain strong positive relationships with all of those involved in the process, taking into account individual and institutional motivations and values. We felt that our role was often one of uncovering, interpreting and translating between different types of expertise (Facer and Enright, 2016).

\section{Engaging with arts practice}

Co-produced research often draws on diverse methodologies and ways of producing knowledge. Artists and arts-based methods are often included in co-produced research to open dialogue and support stronger co-production by providing a way for people with different areas of expertise to talk to one another (Beebeejaun et al., 2014). Evans and Piccini (2017: 99) write that the arts can be viewed as 'mediating praxes that translate and transmit knowledge between "researcher" and "communities". The arts can be employed as an engagement tool, a method of research or analysis, as well as a method for dissemination.

Across Productive Margins, we employed a range of creative and arts-based methods, including poetry, playwriting, co-writing a novel, arts installations, songs, photography and devising a game. There are a myriad of creative and arts-based methods, and defining what is meant by this phrase can be problematic. Over the last hundred years, diverse disciplines have repeatedly used and reported arts-based methods and techniques in research (Prosser, 1998). According to Blackstone et al. (2008: 2), 'The attraction of this mode of research derives in part from its widely regarded capacity to directly engage broad sections of the public', hence its popular use in community-based research.

Reflecting on our experiences across the programme, we felt that arts methods were employed with varying results. On some projects, we felt that artists were brought in to 'do the arts bit' and were not viewed as members of the team. In most projects, 
artists did not attend working group meetings or forums, meaning that they seemed peripheral to Productive Margins as a whole. Few artists were involved in projects from beginning to end. In some cases, research associates felt that this had been problematic. In one project where arts practice was central to the research design, the artists were recruited after the research associate and community researchers had developed a research plan. Advice from the recruited artist led to the original project design being reconfigured, requiring organizations to alter timings and the funding of activities, somewhat derailing the beginning of the research. The research associate felt that in hindsight the artist should have been recruited earlier in the process. Other research associates disagreed - in several projects, community researchers wanted time to explore ideas and carry out initial research, so that artists could be employed with a clear brief and any arts activity was linked to research findings. This points to dilemmas of who to involve when: should community members be engaged first and take part in artists' recruitment, or should artists be involved with community organizations and academics at the outset? How and when artists are engaged leads to potentially vastly different projects. We felt that a case could be made both for engaging arts practitioners at the beginning of the project and later; the important aspect was that it should be purposeful. For instance, one project that took place at two sites employed artists on one site at the start of the project to develop an exhibition and engage the local community in a conversation about the research topic. The other site employed arts methods at the end of the project to analyse and then disseminate findings. While the purpose of employing arts methods differed between sites, there was a shared sense of clarity regarding why they were employed.

We felt that community co-researchers differed in their interest in arts, within and across projects. Some were resistant to this approach and chose not to engage in some activities, including performances. Others were unsure initially, but found that they enjoyed the process more than expected. Some questioned the appropriateness of arts methods to meaningfully engage with and answer the research questions. A fourth group reported that the engagement with arts methods was the reason for their involvement in the research. This was challenging to manage, and we felt that this was most successfully managed when there was a plurality of methods and outputs. This meant that community co-researchers could choose where and when, as well as how, to engage with arts practice. Some were ready to jump straight in, others needed to understand more about what was being asked of them before committing, and some preferred to consult and advise rather than to participate. Community co-researchers' needs are best met by offering a range of possibilities and ways to engage, with arts practice being one of the options. Again, this relates to ensuring that the experience, interests and motivations of community researchers are understood and met.

Working on a co-produced research project requires being a strong collaborator. Just as community members may need support in engaging with the research process, the same may be true of artists. Artists need to be confident and skilled in collaborative working, and supported in understanding both the subject matter and the research process, including ethical working practices. Some of the artists involved in Productive Margins found the project very challenging, and they found their role confusing and unclear. Other artists found it quite difficult to 'let go' of their control of a project, which we recognize is challenging, but in some cases this led to questions from community co-researchers about how their views were incorporated into artistic outputs. Overall, those artists who had experience of collaborative working in some form were much more able to engage with and understand the research projects. Arts methods were a way of understanding one another, reflecting on experience and talking about what 
we had learnt, not just making something. The process was as important, if not more important, than the outcome.

In our group conversations and interviews, it became clear that we ourselves had very differing views and experience of working with arts-based methods. Some of us enjoyed working with artists and exploring different creative methods, and we have continued and developed this as part of our research practice. Others found this aspect of the project quite challenging and did not feel equipped or prepared for this work. Some questioned the appropriateness of creative methods in relation to research questions, and there were strong feelings from some that the process had to be equal to, but not more important than, the research outcomes. (This perspective was also vocalized by several community partners.) Many of us had very little experience working with arts-based methods prior to Productive Margins, and we felt that we were learning as we went along. We found very few detailed accounts in the literature regarding how arts methods were used in collaborative research, and the difference that the incorporation of arts methods made to research outcomes. This sometimes made it difficult develop plans and ensure that we were carrying out 'good' research. In one project, the research associate felt that the arts-based methods were being accorded some kind of 'epistemic privilege' over other kinds of knowledge and expertise, and that there was less critical analysis of artistic processes and outputs than other methods and outputs.

Overall, we felt that the 'success' of arts-based methods related to: having a clear rationale for employing arts-based methods; the experience, interest and willingness of the community members to engage in arts practice; the artists' experience in collaborative research or socially engaged practice; and the research associates' experience, confidence and knowledge of arts-based methods. We suggest it would be useful for co-produced research projects to describe and evaluate their use of artsbased methods in detail, so that we can develop best practice in the field.

\section{Creating the conditions for co-production}

During our discussions, we noted that we had all come across the use of the term 'serendipity' (meaning something that happens by chance; an unplanned fortunate discovery) to describe aspects of the process of co-production. This was sometimes in relation to who is in the room to co-produce with, or perhaps the way in which decisions led to important moments of discovery in the research process. We wondered if using the term 'serendipity' may reflect the different positions of people involved in coproduction. Academic leads and community organizations may not have been involved in the day-to-day, practical and time-consuming efforts that created a space in which people could come together and co-produce research. Research associates often took a great deal of time and energy to foster relationships and develop communications where we could see intersections in people's ideas and perspectives that might be the site of something useful.

Serendipity is perhaps more to do with creating opportunities where unexpected things can happen, rather than something that will occur through some unspecified form of osmosis. These unexpected things - small encounters such as a conversation after a meeting, anecdotes, snippets of learning, and chance encounters - can lead to a personal or professional resonance opening up unexpected opportunities. These create unintended impacts and instigate what can be large shifts in research endeavours (Pain et al., 2015; Barke, 2017b). This feature of research is built on human interaction, and relies on a great deal of groundwork and planning, which provides a space and opportunity for such an encounter - these are not passive moments. 
Facer and Enright (2016) identify two 'core problems' relating to the concept of serendipity in co-produced research. The first relates to groups encountering one another in the first place, and the second to the potential challenge of speaking across different cultures and life experiences to find resonance.

We suggest that the first challenge can in part be met through recognizing the importance of who is involved in a co-produced research project. People do not just come together; time needs to be built into projects to allow for the recruitment of community co-researchers. Careful attention should be paid to who to engage with and how. Ideally, research associates need to work closely with community development workers. Roles regarding recruitment should be addressed when projects are developed, and time and money should be specifically allocated to this. On one project, the research associate began the recruitment process by immersing herself in the local community, attending events and talks, visiting existing groups and meeting with people who were active in the local community. This was supported by the community organization with which she was working, and she was given advice, as well as the time and space to carry this out. This led to recruiting a group of co-researchers with a diverse range of skills and experience, most of whom had not previously engaged with research or the community organization before. This process also supported the development of relationships across the community with individuals, groups and professional organizations who would support the project throughout its lifetime, and beyond. This work meant that the project was embedded in the community, and once the funding and university engagement was completed, the project was able to continue, supported by the local community.

With regard to the second problem identified by Facer and Enright (2016), there are certainly challenges inherent in working across diverse cultures and life experiences. These challenges appear to relate to fostering connections when there are fewer points of connection. Addressing this involves creating empathetic spaces, supporting individuals and the development of relationships that respect diversity. One way in which many of us addressed this issue was by developing and facilitating group sessions and discussions that encouraged co-researchers to talk about their experiences and why they wanted to be involved with the project. We discussed the importance of respect and valuing different opinions. Providing a space for this was in the forefront of our minds, and was a central part of planning sessions. We also all noted that while working with a group of co-researchers was important, we also spent a great deal of time developing individual relationships with group members. We were available before and after group meetings, and we regularly checked in with community co-researchers to ensure that they were happy with the project and to see whether they needed additional support. These conversations allowed us to explore the diversity within the group, and to ensure that different opinions and interests were reflected in our projects.

We agree with Pain et al. (2015: 7) that 'Serendipity is not just about chance; there are conditions that underpin serendipity which can be fostered'. Yet accounts as to how serendipity in co-production is nurtured or fostered into existence are notably lacking in any substantial detail.

\section{Conclusion}

It can be particularly challenging on a co-produced research project to find the time and space to be critical and reflexive. We are grateful that Productive Margins provided time and funding for us to come together and reflect on our experiences. 
While we uncovered a great deal of diversity in our approaches and thoughts about co-production, we also found commonality in the themes discussed in this paper. Reflecting across our experience, and writing this paper, has led us to consider what we learnt from working on Productive Margins and what we would put in place in future co-produced research projects.

We decided on these priorities:

1. Have conversations as early as possible with all partners regarding institutional regulations, systems and project timelines. We would then write a project protocol that all collaborators can work with as a starting point. We would keep this updated, and log any changes to protocol in a shared document that all can access.

2. Establish communication rules: how do people stay in touch, how often and through which methods?

3. Recognize thatworking across difference should involve acknowledging difference positively. Collaborators should explicitly discuss roles and responsibilities, drawing out why different individuals and organizations are involved. This is both a pragmatic conversation and a way to highlight and explore power dynamics.

4. Design the training of community co-researchers as a sharing experience that starts by exploring the skills and expertise of community researchers, and then supports everyone involved coming to a collective understanding of the project and an agreed way of working.

5. Consider carefully how, when and why arts-based methods are employed; reflect on their purpose and evaluate their effectiveness related to this, and record unintended impacts.

6. Take time to develop and maintain strong positive relationships with all of those involved in the process, individually and collectively. Build in time for informal and off-task conversations.

To address some of the challenges that can be involved in co-producing research, we call for greater reflexivity and detailed accounts of how co-produced project research groups are developed and supported. We suggest that for this exciting area of research to develop we, as practitioners, need to share our success and failures and open a positive and critical reflexive space. We hope that through describing the methods employed in co-production, and reflecting on their effectiveness in an open and critical dialogue, we can develop adaptive and robust methodologies that help us to work across difference and create the conditions for co-production.

\section{Acknowledgements}

The authors all worked on the Productive Margins: Regulating for Engagement research programme in different capacities. We would like to thank Productive Margins for the funding, time, encouragement and support to write this paper.

\section{Notes on the contributors}

Jenny Barke is a senior research associate in the Department of History, University of Bristol. Jenny's research focuses on the methods and ethics of co-producing research with people and groups outside the university. Her research explores how to engage with, support and train community researchers, and considers how new knowledge is produced through collaborative research. Much of her work has drawn on creative and arts-based methods in collaboration with writers, actors, visual artists and directors. 
Helen Thomas-Hughes is a senior lecturer and director of part-time studies in the University of Bristol's Department of English. Helen's research is focused on coproductive methodologies in the UK, particularly: working with and training community co-researchers; community-engaged pedagogy in higher education within the UK context; and collaborative approaches to designing and delivering parenting and family interventions. Helen spent more than a decade leading and delivering parenting, domestic violence and education-focused interventions in community settings.

Marilyn Howard is an honorary research associate and doctoral student at the University of Bristol, where she worked on the life chances and regulation projects within Productive Margins. Previously she worked in the women's and disability sectors in policy and research roles, as well as in central and local government. Her experience also includes community social work and welfare rights advice. Her research interests include social security, equality, disability and participative approaches.

\section{References}

Armstrong, F. and Alsop, A. (2010) 'Debate: Co-production can contribute to research impact in the social sciences'. Public Money and Management, 30 (4), 208-10. Online. https://doi.org/10.1080/ 09540962.2010 .492178

Banks, S., Armstrong, A., Booth, M., Brown, G., Carter, K., Clarkson, M., Corner, L., Genus, A., Gilroy, R., Henfrey, T., Hudson, K., Jenner, A., Moss, R., Roddy, D. and Russell, A. (2014) 'Using co-inquiry to study co-inquiry: Community-university perspectives on research'. Journal of Community Engagement and Scholarship, 7 (1), 37-47.

Barke, J. (2017a) 'Community-based research and approaches to loneliness prevention'. Working with Older People, 21 (2), 115-23. Online. https://doi.org/10.1108/WWOP-10-2016-0032

Barke, J. (2017b) Isolation and Loneliness of Older People: Coproducing research with community researchers. Bristol: University of Bristol.

Beebeejaun, Y., Durose, C., Rees, J., Richardson, J. and Richardson, L. (2014) '“'Beyond text": Exploring ethos and method in co-producing research with communities'. Community Development Journal, 49 (1), 37-53. Online. https://doi.org/10.1093/cdj/bst008

Beebeejaun, Y., Durose, C., Rees, J., Richardson, J. and Richardson, L. (2015) 'Public harm or public value? Towards coproduction in research with communities'. Environment and Planning C: Government and Policy, 33 (3), 552-65. Online. https://doi.org/10.1068\%2Fc12116

Blackstone, M., Given, L., Levy, J., McGinn, M., O’Neill, P., Palys, T. and Van den Hoonaard, W. (2008) Research Involving Creative Practice: A chapter for inclusion in the TCPS. Ottawa: Interagency Advisory Panel and Secretariat on Research Ethics.

Boynton, P.M. (2002) 'Life on the streets: Experiences of community researchers in a study of prostitution'. Journal of Community and Applied Social Psychology, 12 (1), 1-12. Online. https://doi.org/10.1002/casp.657

Burholt, V., Nash, P., Naylor, D. and Windle, G. (2010) 'Training older volunteers in gerontological research in the United Kingdom: Moving towards an andragogical and emancipatory agenda'. Educational Gerontology, 36 (9), 753-80. Online. https://doi.org/10.1080/03601271003766270

Cohen, S., Cole, T., McDermont, M. and Piccini, A. (2020) 'Co-production as experimentation: The research forum as method'. In McDermont, M., Cole, T., Newman, J. and Piccini, A. (eds) Imagining Regulation Differently: Co-creating for engagement. Bristol: Policy Press, 23-41.

Enright, B. and Facer, K. (2017) 'Developing reflexive identities through collaborative, interdisciplinary and precarious work: The experience of early career researchers'. Globalisation, Societies and Education, 15 (5), 621-34. Online. https://doi.org/10.1080/14767724.2016.1199319

Evans, P. and Piccini, A. (2017) 'The regulatory aesthetics of co-production'. In Ersoy, A. (ed.) The Impact of Co-Production: From community engagement to social justice. Bristol: Policy Press, 99-118.

Facer, K. and Enright, B. (2016) Creating Living Knowledge: The Connected Communities Programme, community-university relationships and the participatory turn in the production of knowledge. Bristol: University of Bristol and AHRC Connected Communities Programme.

Heron, J. and Reason, P. (2008) 'Extending epistemology within a co-operative inquiry'. In Reason, P. and Bradbury, H. (eds) The SAGE Handbook of Action Research: Participative inquiry and practice. 2nd ed. London: SAGE Publications, 366-80. 
Howard, M., McDermont, M. and Innes, M. (2020) 'Introduction: From the regulation of engagement to regulating for engagement'. In McDermont, M., Cole, T., Newman, J. and Piccini, A. (eds) Imagining Regulation Differently: Co-creating for engagement. Bristol: Policy Press, 1-22.

Howard, M. and Thomas-Hughes, H. (2020) 'Conceptualising quality in co-produced research'. Qualitative Research. Online. https://doi.org/10.1177/1468794120919092

McDermont, M., Cole, T., Newman, J. and Piccini, A. (eds) (2020) Imagining Regulation Differently: Co-creating for engagement. Bristol: Policy Press.

Pain, R., Askins, K., Banks, S., Cook, T., Crawford, G., Crookes, L., Darby, S., Heslop, J., Holden, A., Houston, M., Jeffes, J., Lambert, Z., McGlen, L., McGlynn, C., Ozga, J., Raynor, R., Robinson, Y., Shaw, S., Stewart, C. and Vanderhoven, D. (2015) Mapping Alternative Impact: Alternative approaches to impact from co-produced research. Durham: Durham University.

Pohl, C., Rist, R., Zimmermann, A., Fry, P., Gurung, G.S., Schneider, F., Speranza, C.I., Kiteme, B., Boillat, S., Serrano, E., Hirsch Hadorn, G. and Weismann, U. (2010) 'Researchers' roles in knowledge co-production: Experience from sustainability research in Kenya, Switzerland, Bolivia and Nepal'. Science and Public Policy, 37 (4), 267-81. Online. https://doi.org/10.3152/030234210X496628

Porter, L. (2010) Unlearning the Colonial Cultures of Planning. Farnham: Ashgate Publishing.

Prosser, J. (ed.) (1998) Image-Based Research: A sourcebook for qualitative researchers. London: Falmer Press.

Thomas-Hughes, H. (2018) 'Ethical "mess" in co-produced research: Reflections from a UK-based case study'. International Journal of Social Research Methodology, 21 (2), 231-42. Online. https://doi.org/10.1080/13645579.2017.1364065

Thomas-Hughes, H. and Barke, J. (2018) Community Researchers and Community Researcher Training: Reflections from the UK's Productive Margins: Regulating for Engagement programme (Law Research Paper 10). Bristol: University of Bristol. 